\title{
Evaluation of stroke related risk factors in wake up and non-wake up stroke patients
}

\author{
Elyar Sadeghi-Hokmabadi, ${ }^{1}$ Samad Shams Vahdati, ${ }^{2}$ Reza Rikhtegar, ${ }^{1}$ Nazila Karzad, ${ }^{1}$ \\ Aysa Rezabakhsh ${ }^{3}$
}

${ }^{1}$ Neurosciences Research Center (NSRC), Tabriz University of Medical Sciences; ${ }^{2}$ Emergency Medicine

Research Team, Tabriz University of Medical Sciences; ${ }^{3}$ Cardiovascular Research Center, Tabriz University of Medical Sciences, Tabriz, Iran

\begin{abstract}
Wake-Up Stroke (WUS) is defined as a stroke with the unclear onset of symptoms and subsequent neurological deficits which perceived upon awakening. WUS patients are often excluded from acute fibrinolytic and reperfusion therapy due to the unknown exact time of symptoms onset. This study aimed to evaluate patients with and without WUS characteristics and associated risk factors at two tertiary hospitals. First, we prospectively evaluated consecutive patients with stroke symptoms and determined stroke sub groups by using Computed Tomography (CT) scan. Next, demographic and clinical characteristics including past medical and drug consumption history as well as cardiac function index (ejection fraction), LDL ( $\mathrm{mg} / \mathrm{dl})$ level and hematologic parameters: hemoglobin $(\mathrm{Hb})$; hematocrit $(\mathrm{Hct})$; platelet $(\mathrm{Plt})$ were assessed. Results: 510 patients $(56.1 \%$ men and $43.9 \%$ women) with averaged age of 70 and 72 years were studied, respectively. Of 510 patients, 405 (79.4\%) had non-WUS stroke (known-onset stroke)
\end{abstract}

\footnotetext{
Correspondence: Aysa Rezabakhsh,Cardiovascular Research Center, Tabriz University of Medical Sciences, Tabriz, Iran.

E-mail: aysapharma.rezabakhsh@gmail.com

Tel.:+98-4133352078 - Fax:+98-4136581558

Key words: Wake Up Stroke; Risk Factors; Familiar History; Hematologic Characteristics.

Conflict of interest All authors declare there is no conflict of interest regarding the article publication.

Contributions: ESH analyzed and interpreted the data; SSV conceived and designed the experiments; RR contributed reagents, materials, analysis tools or data; NK performed the experiments; AR wrote the paper.

Ethics approval and consent to participate: The manuscript does not contain any individual person's data in any form.

Received for publication: 19 January 2020

Accepted for publication: 27 May 2020.

This work is licensed under a Creative Commons Attribution 4.0 License (by-nc 4.0).

${ }^{\circ}$ Copyright: the Author(s), 2020

Licensee PAGEPress, Italy

Emergency Care Journal 2020; 16:8834

doi:10.4081/ecj.2020.8834
}

and 105 (20.6\%) had WUS strokes (unknown-onset stroke). The WUS occurrence most likely was observed in ischemic stroke compared to hemorrhagic one. No significant differences were found between patients from both groups regarding stroke risk factors. However, hypertension and family history were more common in patients with WUS ( $\mathrm{p}>0.05$ ). Moreover, individuals with a previous cerebrovascular accident in WUS group were almost similar to non-WUS counterparts. No differences also detected in case of hematologic characteristics, heart function index and LDL levels between study groups $(\mathrm{p}>0.05)$. Together, wake-up stroke occurs in approximately $20 \%$ of stroke subjects. In this study, patients with WUS had more hypertension and family history.

\section{Introduction}

Stroke or Cerebrovascular Accident (CVA) is a well-known medical emergency with high mortality rates conceived as the second leading cause of death and disability worldwide. ${ }^{1}$ Long-term and serious disability are important outcomes in patients with stroke. ${ }^{2}$ Two main broad categories of stroke include ischemic and hemorrhagic strokes which have somewhat different etiology, clinical course and treatment strategies. ${ }^{3}$ The recent epidemiologic study revealed that ischemic stroke is more common compared to hemorrhagic type. In 2013, around 6.9 million people had an ischemic stroke while 3.4 million people had a hemorrhagic stroke. ${ }^{4}$ Hemorrhagic stroke is also divided into two sub-types including Intracranial Hemorrhage (ICH) and Subarachnoid Hemorrhage (SAH). ${ }^{5}$ Although there is evidence-based development in acute stroke therapy as well as organized care in stroke units, determination of onset time is a critical step in stroke management. ${ }^{6}$ Notably, the onset of stroke symptoms is a vital determinant of eligibility for receiving thrombolytic therapy. ${ }^{7}$ However, certain beginning time of stroke cannot be determined particularly in patients who develop stroke during sleeping and they often awaken with neurologic deficits such as aphasia and decreased consciousness. ${ }^{8}$ This type of stroke is known as Wake Up Stroke (WUS) or unclear-onset stroke. ${ }^{9}$ It has been estimated that WUS approximately accounts for $25 \%$ of all stroke incidence. ${ }^{10,11}$ However, the big challenge in WUS treatment is the time interval between stroke onset, hospital arrival and subsequent limited interventions. As t-PA (tissue-plasminogen activator) is a time-dependent therapeutic strategy that should be performed till 3 to 4.5 hours following stroke symptoms onset, patients with WUS have not been included in thrombolytic treatment due to a higher risk of probable hemorrhagic complications. ${ }^{12}$ However, a subset of these patients received empirical or reperfusion therapy based on imaging criteria. ${ }^{13}$ In the present study, we sought to evaluate WUS prevalence and related effective factors in patients who referred to 
emergency department of Imam-Reza and Razi hospitals, affiliated to Tabriz University of Medical Sciences.

\section{Materials and Methods}

\section{Study Sample and design}

We included all consecutive patients in our cross-sectional study with stroke or stroke-related symptoms who referred to Imam-Reza and Razi hospitals emergency department from AprilMay 2015 until April-May 2016. The stroke patients were classified to WUS and non-WUS groups. Moreover, the patients who received anti-hypertension drugs (controlled hypertension) or without any hypertension history were included while the patients without brain organic lesion (such as seizure, electrolyte imbalance, infections, etc.) as well as the patients who had un-controlled hypertension (without receiving any anti-hypertension medications) were excluded from the study.

\section{Discrimination of stroke subtypes}

CT scan is the first neuro-imaging technique used to identify patients with suspected ischemic or hemorrhagic strokes. These subtypes of strokes were determined by CT scan and the ratio of ischemic and hemorrhagic strokes was precisely estimated. Based on CT scan findings, hyperintensity appearance in cerebrovascular was detected at $\mathrm{SAH}$ and $\mathrm{ICH}$ while these features were not seen in an ischemic stroke condition.

\section{Comparison of stroke-related risk factors}

In the present study, different risk factors related to the stroke occurrence such as hypertension, hyperlipidemia, diabetes mellitus, smoking, alcohol consumption as well as drug history was evaluated by appropriate designed questioner and then comparison was conducted between WUS and non-WUS groups.

\section{Determination of hematologic profiles and a cardiac function index}

By using IDEXX ProCyte Dx ${ }^{\circledR}$ hematology analyzer, blood parameters including levels of $\mathrm{Hb}$, Hct and Plt were measured. Moreover, low-density lipoprotein cholesterol (LDL-C), as one of the key biomarker for hyperlipidemia, was measured with a commercial kit according to the manufacturer's introduction (Cat no: PT10041, Pars Azmun Co; Iran). Also, the cardiac ejection fraction was evaluated by echocardiography imaging under supervision of an expert cardiologist.

\section{Statistical analysis}

After data collection, we used SPSS software ver.17.0.1 (SPSS Inc., Chicago, IL, USA) for statistical analysis. For a description of demographic variables, mean \pm Standard Deviation (SD) and frequency percent were used. To analyze differences between quantitative values, we used the independent $t$-test. Qualitative values also were analyzed by Chi-square or Mann-Whitney U test when appropriated. $\mathrm{P}$ value $<0.05$ was considered statistically significant.

\section{Ethical considerations}

Ethical approval for the current study was achieved from Faculty of Medicine of Tabriz University of Medical Sciences with ref number of 93/1-9/17 and ethical code of 5/4/10687. Informed consent was obtained from all individuals participated in the current study.

\section{Results}

Of 510 subjects evaluated during the timeframe of the study (one year), 316 and 194 patients from Imam Reza and Razi hospital emergency department were included, respectively. Of the total 510 patients, $405(79.4 \%$ ) had non-WUS (known-onset stroke) and $105(20.6 \%)$ had WUS (unclear-onset stroke). It seems that nonWUS is more common compared to the WUS $(p<0.05$, Table 1). According to our results, the minimum and maximum ages for WUS were 35 and 81 years old, and the calculated median reached 72 years old. While the minimum and maximum age for non-WUS patients were 17 and 92 years old with median of 70 years old. Interestingly, within the WUS candidates, 59 patients $(56.19 \%)$ were male and $46(43.8 \%)$ were female. Similar results were also obtained from non-WUS group. The number of male and female patients were 227 (56.04\%) and 178 (43.95\%), respectively. In this study, the percentage of ischemic stroke incidence was $85.29 \%$. As our data showed, there was no significant differences in demographic characteristics such as age and gender $(p>0.05$, Table 1). As shown in Table 1, there are also no significant differences regarding the previous CVA in the WUS and non-WUS ( $p=0.66$; Table 1). Regarding stroke sub-types, 333 patients (76.6\%) of nonWUS and 102 patients (23.4\%) of WUS caused by ischemic attacks (the number of total patients was 435) $(\mathrm{p}<0.001$, Table 2). There were no remarkable differences between two genders. In the case of hemorrhagic stroke sub-type (the number of total patients were 75$), 66$ patients $(97.1 \%)$ of non-WUS and 2 patients $(2.9 \%)$ of WUS had ICH $(\mathrm{p}<0.001$, Table 2$)$ while 6 patients $(85.7 \%)$ of non-WUS and one patient (14.3\%) of WUS showed SAH $(p<0.001$, Table 2$)$. Because of the relatively small size of the subarachnoid population, $p$-value was not significant but the occurrence of SAH in females was more than male patients. Previous CVA was considered as one of the key risk factors for stroke incidence. Table 3 exhibited well-established risk factors involved in stroke occurrence. As shown, 388 patients $(76.1 \%)$ had high blood pressure experience in their medical history (Table 3). Moreover, $117(22.9 \%), 77(15.1 \%)$ and $48(9.4 \%)$ patients had ischemic heart diseases, atrial fibrillation, and heart failure, respectively. It seems that the history of cardiovascular disorders plays an important role in subsequent stroke incidence. Other important risk factors in the occurrence of stroke are hyperlipidemia and diabetes mellitus (as a metabolic disorder). Our data showed 175 (34.3\%) and $143(28 \%)$ of patients had hyperlipidemia and type 1 diabetes, respectively. According to our results, smoking and alcohol consumption had a lower role as stroke risk factors. However, 105 patients $(20.6 \%)$ had a smoking history in their life span and just 9 patients $(1.8 \%)$ were alcohol consumers. According to recent data, there were no statistically significant differences between the two groups ( $p>0.05$; Table 3 ). However, the percentage of hypertension and familiar history risk factors in the occurrence of WUS were higher than non-WUS (hypertension: 77.03 vs. 72.38, $\mathrm{p}=0.37$; familiar history: 12.34 vs. $6.8, \mathrm{p}=0.13$ ). In the next step, we evaluated the effect of antiplatelet, anti-coagulants and contraceptive drugs consumption. Our results showed no significant differences between non-WUS and WUS in relation to drug consumption ( $p>0.05$; Table 3$)$. As outlined in Table 4, neither hematologic characteristics (Hb, Ht, Plt) nor cardiac function index (EF) and LDL as lipid profile did not differ significantly between two groups ( $p>0.05$; Table 4$)$. 


\section{Discussion}

In the present study, we found that $20 \%$ of included patients had WUS. Following CT scan imaging to determine stroke subtypes, demographic characteristics and related risk factors analyses were done. Next, important hematologic items along with heart function index and LDL-C were measured as well. Due to WUS importance, the main purpose of this study was the determination of WUS prevalence and major leading risk factors in patients who hospitalized at two tertiary hospitals. In line with previous studies, there are minor demographic and clinical differences between patients with WUS and non-WUS. ${ }^{14,15}$ Controversially, other studies demonstrated that WUS had greater severity at admission and poor prognosis at discharge. ${ }^{16}$ First, our findings showed both known-onset and WUS had almost the same prevalence in men and women and its occurrence most likely observed at older ages (average age: 70-72 years old). In accordance with our results, it has been reported that the average age of stroke occurrence was 67.58

Table 1. Comparison of Demographic Characteristics and Cerebrovascular Accident History in in Patients with Non-Wake Up and Wake Up Strokes.

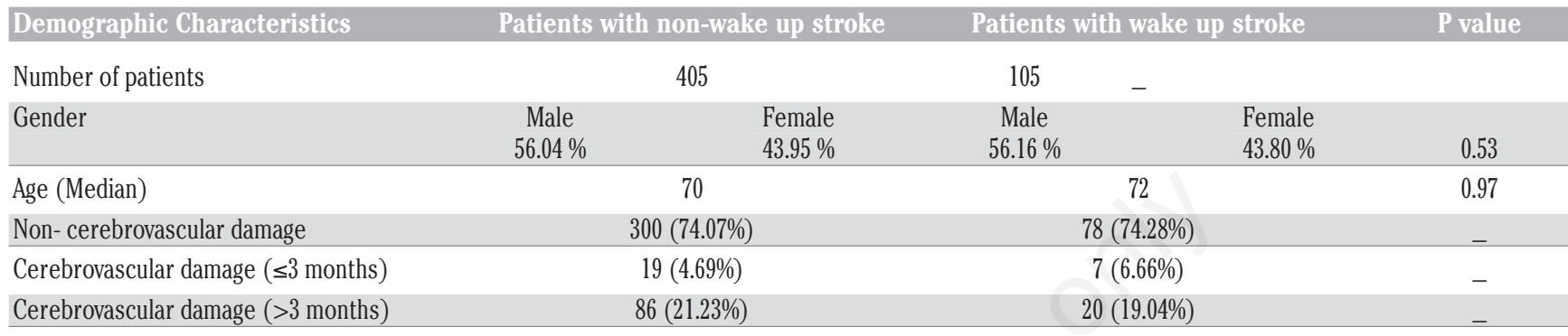

Table 2. Comparison of Stroke Sub -types in Patients with Non-Wake Up and Wake Up Strokes.

\begin{tabular}{|c|c|c|c|c|c|}
\hline Stroke sub- types & & Total & Patients with non-wake up stroke & Patients with wake up stroke & P value \\
\hline & Male (\%) & Female(\%) & & & \\
\hline Ischemic & & 435 & $333(76.6 \%)$ & $102(23.4 \%)$ & $<0.001$ \\
\hline & $57.7 \%$ & $42.3 \%$ & & & \\
\hline $\begin{array}{l}\text { Intracranial } \\
\text { Hemorrhage (ICH) }\end{array}$ & $48.5 \%$ & $51.5 \%$ & $66(97.1 \%)$ & $2(2.9 \%)$ & $<0.001$ \\
\hline $\begin{array}{l}\text { Sub-Arachnoid } \\
\text { Hemorrhage } \\
\text { (SAH) }\end{array}$ & $28.6 \%$ & $71.4 \%$ & $6(85.7 \%)$ & $1(14.3 \%)$ & $<0.001$ \\
\hline
\end{tabular}

Table 3. Comparison of Past Medical and Social History in Patients with Non-Wake Up and Wake Up Strokes.

\begin{tabular}{lccc} 
Past medical and social history & Patients with non-wake up stroke (\%) & Patients with wake up stroke (\%) & P value \\
High blood pressure (hypertension) & 72.38 & 77.03 & 0.37 \\
Diabetes mellitus type 1 & 35.23 & 26.17 & 0.06 \\
\hline Atrial fibrillation & 16.19 & 14.81 & 0.76 \\
Smoking & 21.9 & 20.24 & 0.83 \\
\hline Alcohol consumption & 3.8 & 1.23 & 0.09 \\
Family history & 6.8 & 2.34 & 0.136 \\
\hline Anti- platelet & 31.42 & 6.91 & 0.46 \\
Anti- coagulants & 2.85 & 0.98 & 0.17 \\
\hline Contraceptives & 0 & 0.58 \\
\hline
\end{tabular}

Table 4. Comparison of Hematologic parameters and Heart Function Index in Patients with Non-Wake up and Wake up Strokes.

\begin{tabular}{lcc} 
Hematologic parameters and Heart Function Index & Patients with non-wake up stroke & Patients with wake up stroke \\
Levels of Hemoglobin (Hb) & $13.92 \pm 5.93$ & $13.5 \pm 1.98$ \\
Levels of Hematocrit (Hct) & $41.31 \pm 5.11$ & $40.89 \pm 5.19$ \\
\hline Platelet (Plt) & $257480 \pm 102036$ & $246500 \pm 82700$ \\
Low-Density Lipoproteins (LDL, $\mathrm{mg} / \mathrm{dl})$ & $102.1 \pm 33.71$ & $102.8 \pm 33.15$ \\
\hline Heart ejection fraction & $49.39 \pm 8.59$ & $48.8 \pm 8.49$ \\
\hline
\end{tabular}


\pm 15.2 and $68.88 \pm 15.4 \%$ in known and unknown onset strokes, respectively. ${ }^{17}$ Although the risk of stroke occurrence increases with aging ( $\geq 65$ years old), our results showed that it could be rarely seen in early ages (non-WUS: 35 years old, WUS: 17 years old). It has been proven that the most common sub-type of stroke is an ischemic form which accounts for $87 \%$ of all strokes. ${ }^{18}$ Our results also declared ischemic stroke had a higher occurrence in both WUS and non-WUS patients compared with hemorrhagic sub-type $(\mathrm{p}<0.001)$. Moreover, a large number of clinical findings demonstrated an early-morning high occurrence of ischemic strokes. ${ }^{19,20}$ Albeit, Bornstein et al. reported some risk factors effect on stroke incidence, they could not find significant differences between WUS and non-WUS groups. ${ }^{16,21}$ Recently, a prospective cohort study revealed that there is a reverse relationship between the level of education and stroke occurrence. In better words, lower level of education is tightly associated with increased stroke occurrence thereby it could be considered as possible risk factor of stroke. To note, this association attenuates during older ages but persists. Together, most of the risk factors involved in the stroke can be managed by changing their lifestyle. ${ }^{22}$ Moreover, sleep disorder is another indispensable risk factor which is increasingly associated with stroke. To prevent primary and reoccurrence stroke, it should be address to the sleep disorders before and after stroke. In fact, sleep disorders following stroke can affect related consequence and can interfere in recovery process in the patients. ${ }^{23-25}$ Our data indicated that the number of WUS patients with hypertension was more than non-WUS group. It is noteworthy to mention that cardiovascular disorders play an important role in subsequent stroke incidence. Interestingly, we reported a familiar history supposed another important risk factor. ${ }^{26,27}$ Presumably, it would be helpful to provide prospective treatment protocol to manage WUS patients. We also evaluated the effect of short- and long-term CVA in both subgroups. But appreciable differences were not observed between WUS and non-WUS patients. Furthermore, we observed stroke patients with anticoagulant, antiplatelet or contraceptive drugs history but the significant differences could not be detected between patients with WUS and nonWUS. However, the percentage of patients with anti-platelet consumption was higher than other medications. Although the antiplatelet and anticoagulant therapies are prescribed recently in patients who had prior stroke attacks other protective approaches should be also considered. ${ }^{28}$ Eventually, the hematologic panel along with cardiac ejection fraction and LDL level was evaluated. The results did not show a remarkable increase in WUS rate in comparison with non-WUS subjects. Therefore, hematologic characteristics, cardiac functional indices, and lipid profiles changes are not proper diagnostic criteria for WUS differentiation. Despite less available data in this field, Yilmaz et al. recently demonstrated that the Mean Platelet Volume (MPV) and Platelet Distribution Width (PDW) also had no significant increase in patients with the acute ischemic stroke while the WBC and Neutrophil-Lymphocyte ratio $(\mathrm{N} / \mathrm{L})$ ratio was considerably high in comparison with the control group. Additionally, they noted that WBC and N/L ratio could be helpful for an earlier diagnosis with acute ischemic stroke especially in children..$^{29}$ Based on our data, cardiac ejection fraction ratio was decreased non-significantly in WUS patients. As above-mentioned, cardiovascular disorders history have a critical role in ischemic stroke occurrence. Additionally, it has been suggested that lower left ventricular ejection fraction $(\leq 50 \%)$ in combination with other risk factors such as diabetes mellitus and low level of HDL (high-density lipoprotein) potentially increases stroke risk. ${ }^{30,31}$

\section{Conclusions}

To date, clinical management of WUS is a controversial issue. As the importance of WUS, we aimed to study the prevalence of wake up stroke $(20 \%)$ in patients who referred to the tertiary care hospitals. The results showed that there is an inconsiderable difference between clinical findings and risk factors of both wake-up and known-onset strokes. However, according to our results, genetic history and hypertension categorized as two important wakes-up stroke associated risk factors.

\section{References}

1. Katan M, Luft A. Global burden of stroke. Seminars in neurology. Thieme Medical Publishers, 2018. pp. 208-211.

2. Lo AC, Guarino PD, Richards LG, et al. Robot-assisted therapy for long-term upper-limb impairment after stroke. N Engl J Med 2010;362:1772-83.

3. Amarenco P, Bogousslavsky J, Caplan L, Donnan G, Hennerici M. Classification of stroke subtypes. Cerebrovasc Dis 2009;27:493-501.

4. Vos T, Barber RM, Bell B, et al. Regional, and national incidence, prevalence, and years lived with disability for 301 acute and chronic diseases and injuries in 188 countries, 1990-2013: a systematic analysis for the Global Burden of Disease Study 2013. Lancet 2015;386:743-800.

5. Omama S, Yoshida Y, Ogawa A, Onoda T, Okayama A. Differences in circadian variation of cerebral infarction, intracerebral haemorrhage and subarachnoid haemorrhage by situation at onset. J Neurol Neurosurg Psychiatry 2006;77:1345-9.

6. Powers WJ, Derdeyn CP, Biller J, et al. 2015 American Heart Association/American Stroke Association focused update of the 2013 guidelines for the early management of patients with acute ischemic stroke regarding endovascular treatment: a guideline for healthcare professionals from the American Heart Association/American Stroke Association. Stroke 2015;46:3020-35.

7. Jovin TG, Chamorro A, Cobo E, de Miquel MA, Molina CA, Rovira A, San Román L, Serena J, Abilleira S, Ribó M. Thrombectomy within 8 hours after symptom onset in ischemic stroke. N Engl J Med 2015;372:2296-306.

8. Cucchiara B, Kasner S, Wolk D, et al. Lack of hemispheric dominance for consciousness in acute ischaemic stroke. J Neurol Neurosurg Psychiatry 2003;74:889-92.

9. Kang DW, Kwon JY, Kwon SU, Kim JS. Wake-up or unclearonset strokes: are they waking up to the world of thrombolysis therapy?. Int J Stroke 2012;7:311-20.

10. Mackey J, Kleindorfer D, Sucharew H, et al. Population-based study of wake-up strokes. Neurologym 2011;76:1662-7.

11. Atkinson G, Jones H, Ainslie PN. Circadian variation in the circulatory responses to exercise: relevance to the morning peaks in strokes and cardiac events. Eur J Appl Physiol 2010;108:15-29.

12. Siniscalchi A, De Sarro G, Pacifici R, et al . Thrombolytic Therapy in Cocaine Users with Ischemic Stroke: A Review of Current Practice. Psychopharmacol Bull 2019;49:70-9.

13. Barreto AD, Martin-Schild S, Hallevi H, et al. Thrombolytic therapy for patients who wake-up with stroke. Stroke 2009;40:827-32.

14. Nadeau JO, Fang J, Kapral MK, Silver FL, Hill MD. Outcome after stroke upon awakening. Can J Neurol Sci 2005;32:232-6. 
15. Rimmele D, G. Thomalla G. Wake-up stroke: clinical characteristics, imaging findings, and treatment option-an update. Front Neurol 2014;5:35.

16. Kim BJ, Lee S-H, Shin C-W, et al. Ischemic stroke during sleep: its association with worse early functional outcome. Stroke 2011;42:1901-6.

17. Silva GS, Lima FO, Camargo EC, et al. Wake-up stroke: clinical and neuroimaging characteristics. Cerebrovasc Dis 2010;29:336-42.

18. Béjot Y, Daubail B, Giroud M. Epidemiology of stroke and transient ischemic attacks: Current knowledge and perspectives. Rev Neurol 2016;172:59-68.

19. Kocer A, Ilhan A, Ince N, Bilge C. The related causes in very early morning onset of stroke. Prog Neuropsychopharmacol Biol Psychiatry 2005;29:983-8.

20. Jiménez-Conde J, Ois A, Rodríguez-Campello A, Gomis M, Roquer J. Does sleep protect against ischemic stroke? Less frequent ischemic strokes but more severe ones. J Neurol 2007;254:782-8.

21. Bornstein NM, Gur AY, Fainstein PF, Korczyn AD. Stroke during sleep: epidemiological and clinical features. Cerebrovasc Dis 1999;9:320-2.

22. Jackson CA, Sudlow CLM, Mishra GD. Education, sex and risk of stroke: a prospective cohort study in New South Wales, Australia. BMJ Open 2018;8:e24070.
23. Hepburn M, Bollu PC, French B, Sahota P. Sleep medicine: stroke and sleep. Mo Med 2018;115:527 $\square 32$.

24. Khot SP, Morgenstern LB. Sleep and stroke. Stroke 2019;50:1612-7.

25. Ferrea A, Ribób M, Rodríguez-Lunab D, Romeroa O, Sampolc G, Molinab CA, Álvarez-Sabinb J. Strokes and their relationship with sleep and sleep disorders. Neurología 2013;28:103-18.

26. Seshadri S, Beiser A, Pikula A, et al. Parental occurrence of stroke and risk of stroke in their children: the Framingham study. Circulation 2010;121:1304.

27. Kubota M, Yamaura A, Ono J-i, et al. Is family history an independent risk factor for stroke?. J Neurol Neurosurg Psychiatry 1997;62:66-70.

28. Kapil N, Datta YH, Alakbarova N, et al. Antiplatelet and anticoagulant therapies for prevention of ischemic stroke. Clin Appl Thromb Hemost 2017;23:301-18.

29. Yilmaz E, Kacar AB, Bozpolat A, et al. The relationship between hematological parameters and prognosis of children with acute ischemic stroke. Childs Nerv Syst 2018;34:655-61.

30. Pullicino P, Raynor S. Is low cardiac ejection fraction a risk factor for stroke?. Malta Med J 2013;25:10-7.

31. Fidha Rahmayani P, Setyopranoto I. The role of ejection fraction to clinical outcome of acute ischemic stroke patients. J Neurosci Rural Pract 2018;9:197-202. 